\title{
Azobenzene: a visible-light chemical actinometer for the characterization of fluidic photosystems
}

\author{
Mélanie Roseau, ${ }^{a}$ De Waele Vincent, ${ }^{b}$ Xavier Trivelli, ${ }^{c}$ Francois Xavier Cantrelle, ${ }^{\text {d,e }}$ Maël Penhoat, ${ }^{a}$ and Laëtitia \\ Chausset-Boissarie ${ }^{* a}$ \\ a USR 3290 MSAP, Lille University, CNRS, Villeneuve d'Ascq, France, Laetitia.boissarie@univ-lille.fr \\ b UMR 8516, LASIR, Lille University, Villeneuve d'Ascq, France \\ c FR 2638 IMEC, Lille University, CNRS, INRA, Centrale Lille, Artois University, Lille, France \\ dERL9o02 Integrative Structural Biology, CNRS, Lille, France \\ e U1167 RID-AGE, Lille University, Inserm, CHU Lille, Institut Pasteur Lille, Lille, France \\ Dedicated to Professor E. Peter Kündig on the occasion of his $75^{\text {th }}$ Birthday
}

(E)-Azobenzene is introduced as a suitable chemical actinometer in the visible spectral range (440-540 nm) for photon flux determination of fluidic microphotoreactors or for assessing efficiency of visible light photo-induced reactions, its evaluation is straightforward without tedious analytics. Photoisomerization quantum yields $\left(\Phi_{E} \rightarrow z\right)$ of $(E)$-azobenzene were accurately determined upon irradiation at several wavelengths and in different solvents based on well-known diarylethene.

Keywords: Azobenzene $\bullet$ Actinometry $\bullet$ Visible light $\bullet$ Microfluidic photoreactor

\section{Introduction}

Visible light-promoted photochemical reactions have gained increasing attention enabling elegant bond-disconnection strategies that would otherwise been difficult to achieve. ${ }^{[1-7]}$ Since the last decade, continuous flow photochemistry has emerged as a powerful technique which favors the application of photochemical reactions especially at an industrial level. ${ }^{[8-11]}$ Simple protocols to determine photon flux inside microfluidic systems are crucial for the characterization of photochemical reactors, their maintenance and their dimensioning for scale-up studies to improve process intensification efficiency of photochemical reactions. Indeed, knowing the value of the photon flux for a given photoreaction is essential for photochemical investigations since it governs the photokinetics of the transformation.

Nowadays, physical radiometry is widely used due to its facility of implementation however, the measurement only provides the incident photon flux and not the amount of absorbed photons by the reaction mixture. On the contrary, chemical actinometry allows to determine with a good range of precision the photon flux which crosses the reactive medium under the same experimental conditions of the studied photoreaction. Thus, a chemical actinometer is a photosensitive system which undergoes reversible or irreversible chemical transformation upon irradiation at a specific wavelength proportional to the amount of received photons. ${ }^{[12]}$ To define a new chemical actinometer at a specific wavelength, the quantum yield of the selected transformation for a specific wavelength has to be determined. Numerous actinometers have been developed in the UV domain however, only a few numbers are suitable to study light in the visible region along with severe limitations. Moreover, despite the growing interest for continuous flow processes, actinometric protocols for the determination of photon fluxes in microphotoreactors have been limited. For reliable measurements it is imperative that the conversion rate of the actinometer changes linearly with the irradiation time which is directly quantitatively correlated to the irradiated photon flux. Ideally, to minimize measurement errors it is recommended to work under full absorption conditions for a given irradiation wavelength so all the incident photons are captured during the reaction however, full absorption conditions are difficult to achieve using fluidic microsystems. ${ }^{[13]}$

Aillet et al. ${ }^{[14]}$ measured photon fluxes inside FEP (Fluorinated Ethylene Propylene) capillary microreactors thanks to the benchmark HatchardParker actinometer ${ }^{[15]}$ for an irradiation wavelength ranged between 250 and $500 \mathrm{~nm}$ based on a photo-induced reduction of potassium ferrioxalate in water. Following up, the group of Ziegenbalg accurately determined photon fluxes in multilayer FEP capillary photoreactors with pulsed actinometry measurements. ${ }^{[16]}$ However, clogging of the system at high conversion, difficulty to obtain full absorption condition and the necessity to work under dimmed red light still limited its large implementation. The well-known Reinecke salt ${ }^{[17-19]}$ and meso-diphenylhelianthrene ${ }^{[20]}$ based on respectively photoaquation and photooxidation are communally used in batch nevertheless it implies the use of toxic reactant and tedious manipulations. Another important class of visible light actinometers are thermally-stable photochromic molecules. Fulgide derivatives such as Aberchrome $540^{[21]}$ and trifluoromethyl substituted indolylfulgide developed by Yokoyama et al. ${ }^{[22,23]}$ are standard photochromes but unfortunately they are not commercially available. Lately, Roibu et al. presented a readily implemented methodology for photon flux 
determination of a chip microreactor irradiated with green LEDs (Light Emitting Diodes ${ }^{[24]}$ but this requires the use of costly dithienylethene derivative ${ }^{[25]}$ and on-line UV-Vis spectroscopy needs to be performed under dark conditions.

Azobenzene is a typical molecular photo-switch where upon irradiation a (E/Z)-photoisomerization occurs. It's one of the most popular actinometer in the range $245-440 \mathrm{~nm}$ since it is inexpensive, not degraded by prolonged irradiation and, the thermal reaction rate is extremely slow at room temperature. ${ }^{[26-33]}$ Indeed, beyond $450 \mathrm{~nm}$, azobenzene has not been recommended because UV-Vis spectroscopic measurements are more difficult and less accurate due to weakly absorbing solution. The only disadvantage of this methodology is that it requires knowledge of the molar absorptivity of both the $E$ and $Z$ isomers at the wavelength of irradiation which is difficult to obtain for the $Z$ form. To reduce the problem caused by the kinetic evaluation method, our group recently developed a very simple chemical actinometric protocol for the characterization of flow microsystems via the photoisomerization of (E)-Azobenzene at $365 \mathrm{~nm}$ and NMR spectroscopy. ${ }^{[34]}$ The benefits of the present method are that concentrated solution can be used and the knowledge of both the molar absorption coefficient value and the quantum of the $(Z)$-isomer at the irradiation wavelength are unnecessary since ${ }^{1} \mathrm{H}$-NMR allows to determine the concentration of $E$ and $Z$ isomers over time. The combination of this actinometic protocol and microreactors is particularly advantageous since their miniaturized dimensions allow a high level of absorption under high concentration condition leading to accurate actinometric procedures even at high photon fluxes or conversions. Based on these advantages herein, we demonstrated that $(E)$-azobenzene is a practical chemical actinometer for wavelengths in the visible region for the determination of photon flux in photomicroreactors under continuous flow conditions. Quantum yield of $(E)$-azobenzene photoisomerisation $\left(\Phi_{E}\right)$ were measured for different wavelengths thanks to diarylethene actinometry. Furthermore, the influence of the solvent was investigated. This work will extend the spectral range of azobenzene making $(E)$-azobenzene a standard actinometer from the UV to visible region.

\section{Results and Discussion}

\section{Photochemical Kinetics}

Generally, reaction kinetics depend on the incident light intensity, concentration of actinometer molecules, molar absorptivity and quantum yield, which is defined as the number of reactions that occur per absorbed photon, at a given light wavelength. The reversible photoisomerization of azobenzene can be described with the following reaction scheme, the thermal isomerization being neglected for the photokinetic calculations since the temperature of the system is controlled:

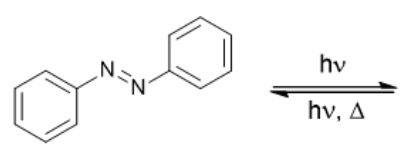

E

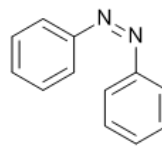

Z
Scheme 1. Photoisomerisation of azobenzene.

Under the assumption that the amount of $(E)-(Z)$ isomerization is proportional to the incident photon number and $Z-E$ back reaction is negligible, quantity of the $(Z)$-azobenzene in the solution is linear to the irradiated photon number. Thus, the overall photon flux received inside a photoreactor can be determined using $(E)$-azobenzene as chemical actinometer considering the following equation.

$$
-\frac{d[E]}{d t}=\frac{d[Z]}{d t}=\Phi L_{A b s}=\frac{I_{A b s}}{V} \Phi(1)
$$

For the $(E \rightarrow Z)$-photoisomerization process, the consumption rate of $(E)$ -

azobenzene under an incident monochromatic light of wavelength $\lambda$, depends on the quantum yield of the photoconversion (mol.ein ${ }^{-1}$ ) and on the average rate of photon absorption by the actinometric solution $L_{A b s}$ (ein. $\mathrm{m}^{-3} \cdot \mathrm{s}^{-1}$ ), which corresponds to the absorbed photon flux $I_{A b s}$ (ein. $\mathrm{s}^{-1}$ ) over the entire irradiated volume of the reactor $V\left(m^{3}\right)$ (eqn 1$)$. In microphotoreactors it is difficult to operate under a total absorption regime, thus the amount of photons absorbed by the mixture of $(E)$ - and (Z)azobenzene in the solution can be expressed via the Beer-Lambert's law where $\Delta A$ is the absorbance of the irradiated solution at a specific wavelength and $l_{0}\left(\right.$ ein. $\left.s^{-1}\right)$ is the overall received incident light intensity of the LED source in the channel (eqn 2).

$$
I_{A b s}=I_{0}\left(1-10^{-\Delta A}\right)(2)
$$

Equation 2 can be simplified using Taylor series expansion of the absorbance

$$
I_{A b s}=I_{0} \ln 10 \Delta A \text { (3) }
$$

Accordingly, the knowledge of $I_{o}$ and the absorbance yields $I_{A b s}$.

The formation rate of $(Z)$-azobenzene excluding the thermal isomerization can then be expressed as follows (a detailed description of the mathematical formulation can be found elsewhere) $)^{33}$ :

$$
\frac{d[Z]}{d t}=\frac{\Phi_{E} I_{0} \ln 10 \varepsilon_{E}(1-z) C_{0} l}{V}-\frac{\Phi_{Z} I_{0} \ln 10 \varepsilon_{Z} z C_{0} l}{V}(4)
$$

Where $z$ is the fraction of $Z$ isomer, (1-z) the fraction of $E$ isomer, $\varepsilon_{E}$ and $\varepsilon_{Z}$ (L. $\mathrm{mol}^{-1} \cdot \mathrm{cm}^{-1}$ ) the molar absorption coefficient of $E$ and $Z$-azobenzene respectively, $C_{o}\left(\mathrm{~mol}^{-L^{-1}}\right)$ the initial concentration, $\Phi_{E}$ and $\Phi_{Z}$ the quantum yield of $E$ and $Z$-azobenzene respectively, $V(L)$ the volume of the reactor and $l(\mathrm{~cm})$ the optical path length.

By considering that at the photostationary state (PSS) $\left(\mathrm{d}\left[Z_{P S S}\right]\right) / \mathrm{dt}=0$, equation (4) can be simplified as:

$$
\frac{d\left[Z_{P S S}-Z\right]}{d t}=\frac{I_{P h o t o n} \Phi_{E} \varepsilon_{E} \ln 10}{Z_{P S S}}\left[Z_{\mathrm{PSS}}-Z\right] \text {, with } I_{P h o t o n}=\frac{I_{0} l}{V}(5)
$$

The integration of this equation will give a linear relationship between $\ln \left[Z_{P S S}-Z\right]$ and the time where the flux density of photon received at the reactor wall $I_{\text {photon }}\left(\right.$ ein. $\mathrm{s}^{-1} \cdot \mathrm{m}^{-2}$ ) as function of the emitted light can be determined directly from the slope of the curve $\mathrm{k}$.

$$
I_{P h o t o n}=\frac{Z_{P S S} k}{\Phi_{E} \varepsilon_{E} \ln 10}(6)
$$

The radiant power per unit of irradiated surface $Q_{\text {inside }}$ expressed in W. $\mathrm{m}^{-2}$ is obtained by multiplying $I_{\text {photon }}$ by $N_{A}$ the Avogadro constant (6.02.1023 $\mathrm{mol}^{-1}$ ) and $\Delta E$ which is the energy of a photon $(J)$, where $h$ the Planck constant $\left(6.6256 .10^{-34} \mathrm{~J} . \mathrm{s}\right) \mathrm{c}$ the speed of light $\left(2.9979 .10^{8} \mathrm{~m} . \mathrm{s}^{-1}\right)$ for the selected irradiation wavelength (eqn 7). 


$$
Q_{\text {inside }}=I_{\text {Photon }} \Delta E N_{A}, \quad \text { with } \Delta E=\frac{h c}{\lambda}(7)
$$

Finally, the irradiance at the surface $Q_{\text {outside }}$ can be calculated from $Q_{\text {inside }}$ and the reactor material transmittance $T$ such as:

$$
Q_{\text {inside }}=T Q_{\text {outside }}(8)
$$

Note that this value is correlated with the data recorded with radiometer.

\section{Determination of $(E)$-azobenzene quantum yield}

The rate of the $(E \rightarrow Z)$-photoisomerization of $(E)$-azobenzene have been thoroughly discussed and quantum yields of the reaction, have been determined in both senses in different solvents, light intensities, concentration and for different wavelengths in the UV/Vis region range up to $450 \mathrm{~nm} .^{[26-33]}$ It has been shown that the quantum yield relies heavily on the wavelength regardless of the concentration. We carefully determined the quantum yield of photoisomerization for $(E)$-azobenzene using FEP tubing microphotoreactors in $n$-hexane, acetonitrile and methanol using blue and green LEDs as light sources.

In this work, pink and yellow commercially available LEDs were also studied however due to respectively their polychromatic character or a large emission band their use for actinometric measurements were discarded for this study. Indeed, when irradiations are carried out at the edge of a band where the molar absorption coefficient varies greatly with the wavelength the accuracy of the measurement is not confirmed and should be averaged, thus limiting the quantum yield value to a specific light source which has no physical meaning.

To obtain the quantum yield of $(E)$-azobenzene in different solvents in the visible region, we firstly determined the overall received photon flux of two light sources at 458 and $524 \mathrm{~nm}$ in two FEP tubing microphotoreactors $\mathrm{R}_{1}$ and R2 (i.d. $=800 \mu \mathrm{m}$, Fig S9 \& S19, SI) of defined geometry via actinometric measurements using the photocycloreversion reaction of the diarylethene derivatives $\mathbf{1}$ or $\mathbf{2}$ from its closed form to the open form following the procedure reported by Irie and coll[25] and revisited by Roibu et al. ${ }^{[24]}$

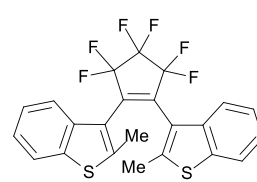

$1 \mathrm{a}$

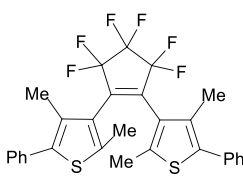

2a
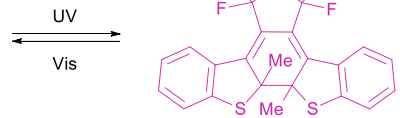

$1 \mathrm{~b}$
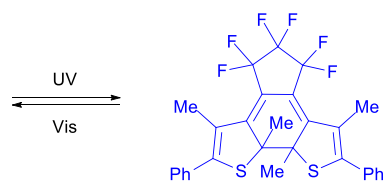

Figure 1. Opened-ring and closed-ring isomers of dithienylethenes $\mathbf{1}$ and $\mathbf{2}$.

Indeed, closed-ring isomers of 1,2-Bis(2,4-dimethyl-5-phenyl-3-thienyl)3,3,4,4,5,5-hexafluoro-1-cyclopentene $\quad 1 b \quad$ and 1,2-Bis[2methylbenzo[b]thiophen-3-yl]-3,3,4,4,5,5-hexafluoro-1-cyclopentene $\mathbf{2 b}$ can be respectively utilized in the visible range between $450 \mathrm{~nm}-580 \mathrm{~nm}$ and $480-620 \mathrm{~nm}$. These colored closed-ring isomers need to be formed under
UV irradiation from the thermodynamically stable colorless open-ring isomers immediately prior to measurements.

The UV-Vis spectrum of the isomerically pure 1 a was recorded prior to irradiation (Fig 3a). Then colorless solution of $\mathbf{1 a}$, dissolved in $n$-hexane (3.6.10-3 mol. $\left.\mathrm{L}^{-1}\right)$ was irradiated with UV lamp at $312 \mathrm{~nm}$ at different times. After each irradiation time, the UV-Vis spectra were measured and used to determine whether the photostationary state (PSS) was reached, (e.g. constant absorbance value at $\left.515 \mathrm{~nm}\left(\lambda_{\max }\right)\right)$ where the $\mathbf{1 a}$ isomer does not absorb (Fig 3a). Under fluidic conditions, the PSS was obtained after only 9 s. Importantly, when the solution is irradiated after reaching the PSS, an unidentified photo by-product is formed leading to a decrease of the visible absorbance (Fig 3b). Thus, to obtain accurate measurements overirradiation must be avoided.
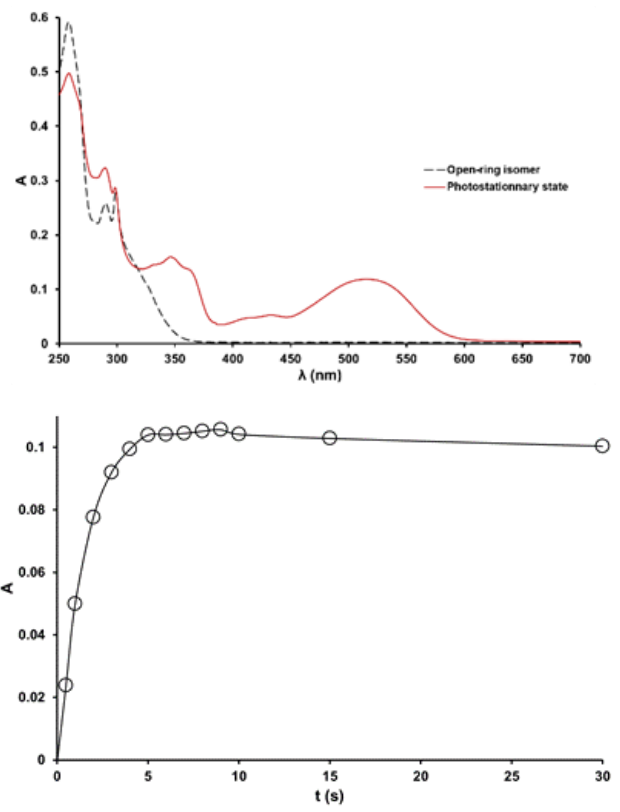

Figure 2. (a) Absorption spectra of 1 (3.6.10-5 mol. $\left.\mathrm{L}^{-1}\right)$ in $n$-hexane. Dashed line: isomerically pure open-ring isomer; red line: photostationary state at $312 \mathrm{~nm}$ under microfluidic conditions. (b) Absorbance at $515 \mathrm{~nm}$ of 1 under irradiation at $312 \mathrm{~nm}$ vs irradiation time (e.g. at various flow rates) under microfluidic conditions. FEP Tubing microphotoreactor (i.d. $=800 \mu \mathrm{m}, \mathrm{l}=21 \mathrm{~cm}, \mathrm{~V}=0.11 \mathrm{~mL}$ ).

The same methodology was repeated for the molecule 2 (Fig $\mathrm{S}_{3}, \mathrm{~S}_{10}, \mathrm{SI}$ ). The conversions from the open to the closed-ring isomers at the PSS determined by NMR spectroscopy were found to be $36 \%$ (Fig S45-47, SI) and $77 \%$ (Fig $\mathrm{S}_{50}-52, \mathrm{SI}$ ) of $\mathbf{1 a}$ and $\mathbf{2 b}$ respectively which is in the same range than that previously reported. ${ }^{[24,25]}$

A typical actinometry experiment with $\mathbf{1 b}$ is described. The irradiated solution of $\mathbf{1 b}$ was injected into the microphotoreactor (FEP Tubing reactor: i.d. $=800 \mu \mathrm{m}, \mathrm{I}=21 \mathrm{~cm}, \mathrm{~V}=0.11 \mathrm{~mL})$, irradiated with blue LED $\left(\lambda_{\max }=458\right.$ $\mathrm{nm}$ ) at various flow rate and the decrease in absorbance at $458 \mathrm{~nm}$ was measured after each residence time interval. The mathematical expression used to determine the photon flux is given by equation (9) (a detailed description of the mathematical formulation can be found in a previous article) ${ }^{[24]}$

$$
\log \left(10^{A_{0}}-1\right)-\log \left(10^{A_{t}}-1\right)=\varepsilon_{1 b} \emptyset_{1 b \rightarrow 1 a} l \frac{I_{0}}{V} t(9)
$$


Where $A_{o}$ and $A_{t}$ are the absorbance of the solution at $458 \mathrm{~nm}, \varepsilon_{1 b}$ the molar absorption coefficient of $\mathbf{1} \mathbf{b}$ at $458 \mathrm{~nm}$, / the average optical path length, ${ }^{[35]}$ $\emptyset_{1 \mathrm{~b} \rightarrow 1 \mathrm{a}}$ the ring opening quantum of $\mathbf{1 b}$ and $V$ the volume of the solution. Considering $y=\log \left(10^{A_{0}}-1\right)-\log \left(10^{A_{t}}-1\right)$ so equation (9) can be written in the form of equation (10)

$$
y=k^{\prime} t \text { with } k^{\prime}=\varepsilon_{1 b} \emptyset_{1 b \rightarrow 1 a} I_{\text {Photon }}(10)
$$

The corresponding graph exhibits a linear relationship with an excellent correlation coefficient where the overall received photon flux can be calculated directly from the slope of the curve $k^{\prime}$ as shown by eqn (11) (Fig $\left.\mathrm{S}_{14}, \mathrm{SI}\right)$.

$$
I_{\text {photon }}=\frac{k^{\prime}}{\varepsilon_{1 b} \emptyset_{1 b \rightarrow 1 a}}(11)
$$

For photon flux calculation the following ring opening quantum yield was applied $\emptyset_{1 b \rightarrow 1 a}=0.312 \mathrm{~mol}$ ein ${ }^{-1}$ at $458 \mathrm{~nm} \cdot{ }^{[25]}$

It is possible to determine the molar absorptivity of $\mathbf{1 b}$ by first either recording the absorption spectrum of the isomerically pure $\mathbf{1 a}$ and at the PSS. Then, the molar ratio of $\mathbf{1 a}$ and $\mathbf{1 b}$ at steady state was determined by NMR spectroscopy (Fig S45-47, SI) which enables to calculate the concentration of $\mathbf{1 b}$. Finally, the molar absorption coefficient of $\mathbf{1} \mathbf{b}$ at $\mathbf{a}$ specific wavelength was determined via classical calibration curve having the absorbance vs concentration (Fig $\left.\mathrm{S}_{5}, \mathrm{SI}\right)$. The same calculations were done for the other LED source at $524 \mathrm{~nm}$ (Fig S6, S8, SI) and the results concerning the absorption coefficients and the photon flux density received at the reactor wall inside FEP tubing microphotoreactors $\mathrm{R}_{1}$ or $\mathrm{R}_{2}$ are summarized in table 1.

Table 1. Results of chemical actinometry as a function of wavelength in FEP tubing

\begin{tabular}{|c|c|c|c|c|c|}
\hline \multirow{2}{*}{ Entry } & \multirow{2}{*}{$\begin{array}{l}\text { Wavelength of } \\
\text { irradiation }(\mathrm{nm})\end{array}$} & \multirow{2}{*}{$\varepsilon_{1 b}^{[c]}$} & \multirow{2}{*}{$\varepsilon_{2 b}^{[d]}$} & \multicolumn{2}{|c|}{$I_{\text {photon }}\left(10^{-3}\right.$ ein. $\left.\cdot \mathrm{s}^{-1} \cdot \mathrm{m}^{-2}\right)$} \\
\hline & & & & $\mathrm{R}_{1}{ }^{[\mathrm{e}]}$ & $\mathbf{R}_{2}{ }^{[f]}$ \\
\hline 1 & $45^{8} \pm 25^{[a]}$ & 4700 & - & 3.303 & - \\
\hline 2 & $524 \pm 30^{[b]}$ & 9650 & 8600 & 1.564 & 1.930 \\
\hline \multicolumn{6}{|c|}{ 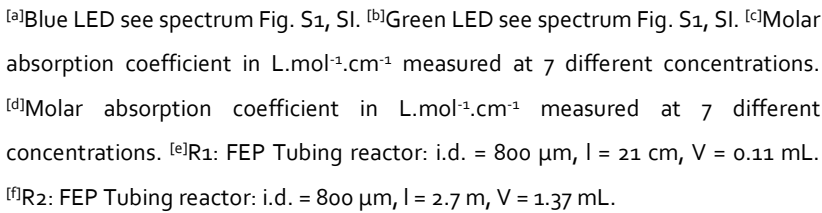 } \\
\hline
\end{tabular}
photoreactors

The irradiance distribution albeit, the overall received photon flux at the wall depends on the distance from the irradiated microphotoreactor to the LED source as well as on the geometry of the irradiated surface (Table 1 , entry 2). Thus, in order to obtain accurate measurements while avoiding modelling of the received irradiance over the photoreactor, identical systems were used during the procedure since the values obtained from $(E)$ -
Azobenzene are correct only if they are measured within the same experimental arrangement.

These data in hand, a solution of $(E)$-Azobenzene in $n$-hexane ( 0.01 moL. $L^{-1}$ ) was injected into FEP tubing microphotoreactor $\mathrm{R}_{1}$ or $\mathrm{R} 2$ and then irradiated at various flow rates by visible LEDs at different wavelength (458 and $524 \mathrm{~nm}$ ) to establish the kinetic data. In order to be able to compare our results with the one previously reported within the $n, \pi^{*}$ absorption bands, the concentrations of $(E)$-Azobenzene were such to ensure the same absorption of the incident light as described by Zimmerman. ${ }^{[33]}$
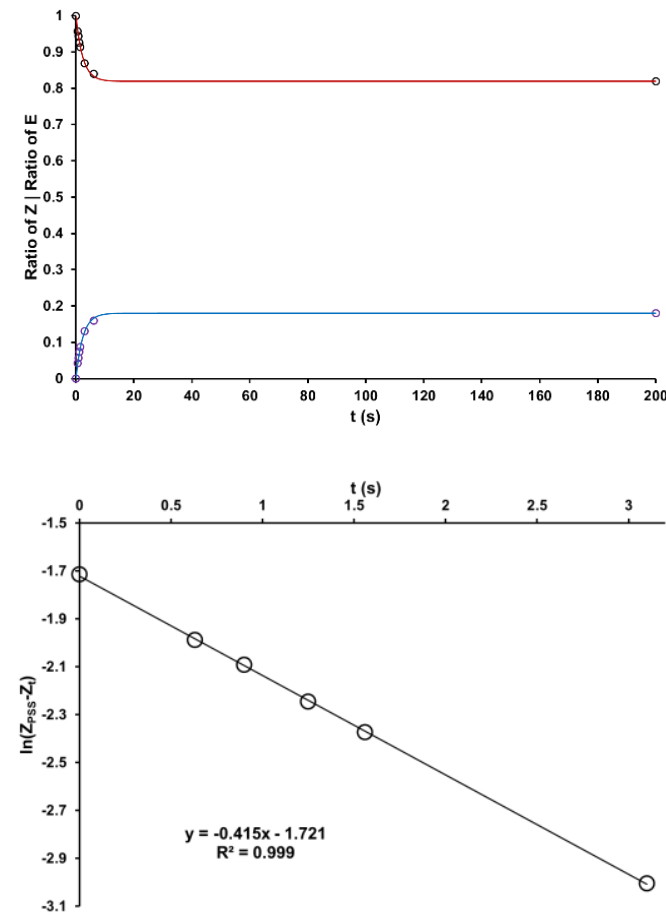

Figure 3. (a) Evolution of $(E)$ and $(Z)$-azobenzene with the irradiation time. (b) Photokinetics study of $(E / Z)$ isomerization of $(E)$-azobenzene at $458 \mathrm{~nm}$ under microfluidic conditions. FEP Tubing reactor (i.d. $=800 \mu \mathrm{m}, \mathrm{I}=21 \mathrm{~cm}, \mathrm{~V}=0.11 \mathrm{~mL}$ ).

The composition of the PSS and the molar extinction coefficient $\varepsilon_{E}$ are independent of the intensity of the incident light or the photoreactor geometry but depends strongly on the wavelength and the nature of the solvent. Thus, the photostationary state composition ( $\left.Z_{P S S}\right)$ and the molar extinction coefficient $\varepsilon_{E}$ were re determined for each wavelength and solvent using respectively NMR and UV-Vis spectrophotometry. For each wavelength, the given data were then used to plot the graph of $\ln \left(Z_{\text {PSS }}-Z\right)$ vs. time which exhibits in each case an excellent squared correlation coefficient $\left(R^{2}>0.99\right)$ (Fig. 4a). Substituting the values of $k^{\prime}\left(0.415 \mathrm{~s}^{-1}\right)$ (Fig. $4 \mathrm{~b})$, which corresponds to the slope of the graph, Zpss (0.18), $\varepsilon_{E}$ (4700 L.mol-1. $\left.\mathrm{cm}^{-1}\right)$ and Iphoton $\left(3 \cdot 303 \cdot 10-3\right.$ ein. $\left.\mathrm{s}^{-1} \cdot \mathrm{m}^{-2}\right)$ in equation 6 the quantum yield of the photo-conversion $\Phi_{E}\left(0.249 \pm 0.01\right.$ mol.ein $\left.^{-1}\right)$ in $n$-hexane is obtained.

The same methodology was applied for the green LED in $n$-hexane (Fig S2730, SI) in $\mathrm{MeOH}$ (Fig S31-33, S37-39, SI) and in acetonitrile (Fig S34-36, S40$42, \mathrm{SI})$. The results of interest are summarized in table 2, the mean values were obtained as an average of three individual runs. 
In non-polar aprotic solvent such as n-hexane, the quantum yield of the $(E \rightarrow Z)$-photoisomerization of $(E)$-azobenzene shows almost no dependence on the irradiation wavelength of excitation within the $n, \pi^{*}$ absorption band in the range $400-530 \mathrm{~nm}$ (Table 2, entries 1, 4). The geometry of the used reactor does not affect the value of the quantum yield but only the value of $I_{\text {photon }}$ (Table 2, entries 4-5). The values in $n$-hexane are in excellent agreement with the data reported in the literature regarding the photoisomerization quantum yields obtained by irradiating in the $n, \pi^{*}$ band at $436 \mathrm{~nm}$ in non-polar solvent. $\left.{ }^{[31,} 33\right]$ The composition of photostationary states depends on the nature of the solvent. Thus, under both irradiation wavelengths the amount of ( $Z$ )-azobenzene isomer increases going from apolar ( $n$-hexane), dipolar aprotic $\left(\mathrm{CH}_{3} \mathrm{CN}\right)$ to dipolar protic $(\mathrm{MeOH})$ solvents. However, the quantum yield of the isomerization was found to be solvent independent (Table 2, entries 1-3, 5-7). (E)azobenzene can thus be used in large range of solvent which is often not the case with other common visible actinometers.

Table 2. Results of chemical actinometry assessment using (E)-Azobenzene in FEP tubing microphotoreactors $\mathrm{R} 1$ or $\mathrm{R} 2$ under visible LEDs

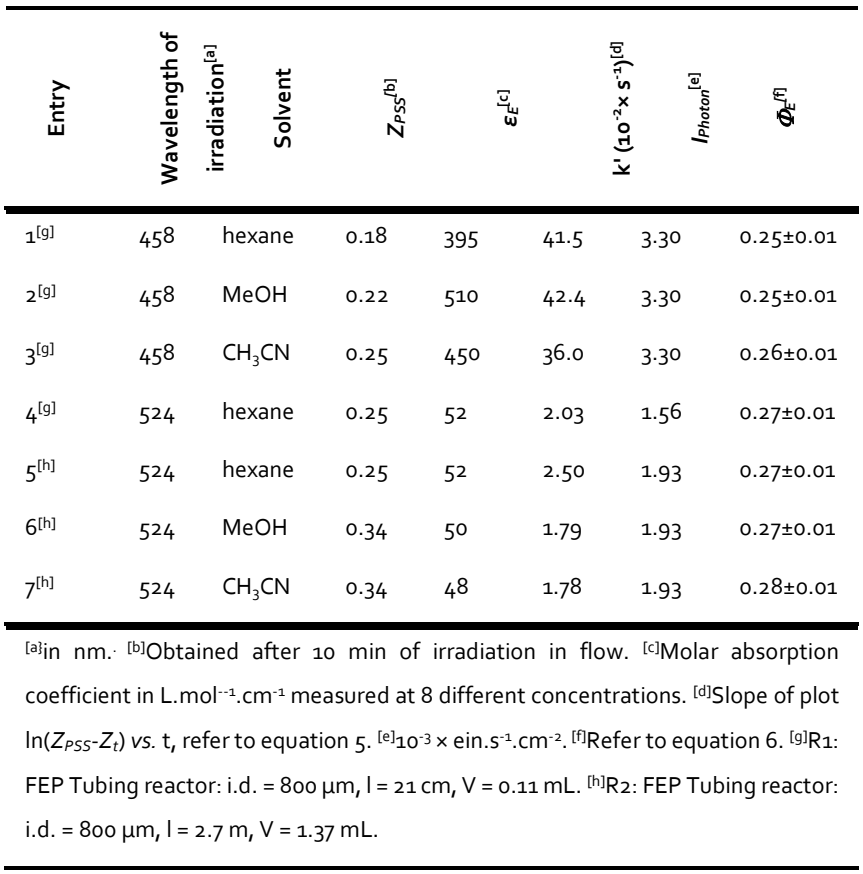

\section{Conclusions}

We determined the quantum yield of the $(E \rightarrow Z)$-photoisomerization of $(E)$ azobenzene in the visible region (440-540 nm) thanks to known dithienylethene derivatives demonstrating that $(E)$-azobenzene is a very convenient actinometer which covers a wide spectral range both in the UV and in the visible region. This actinometric protocol for photo-fluidic microreactor provides the advantage of being much easier to implement compared to other chemical actinometers, allowing to work with high concentration of $(E)$-azobenzene and withstanding longer irradiation times and higher light intensities typically employed in photoredox transformation making it a method of choice for characterizing visible light sources and microphotoreactors.

\section{Experimental Section}

\section{Chemicals and instrumentations}

Reagents were used as received without further purification. (E)Azobenzene (> 98.0\%), 1,2-Bis(2,4-dimethyl-5-phenyl-3-thienyl)$3,3,4,4,5,5$-hexafluoro-1-cyclopentene (purified by sublimation, >99.0\%) 1 and 1,2-Bis[2-methylbenzo[b]thiophen-3-yl]-3,3,4,4,5,5-hexafluoro-1cyclopentene (purified by sublimation, > 99.0\%) 2 were purchased from $\mathrm{TCl}$. $n$-Hexane (> 97\%) and acetonitrile (> 99.9\%) were purchased from Aldrich, methanol (96.5\%) was purchased for VWR Chemicals. Absorption spectra were recorded on a UV-Vis spectrophotometer (Lambda 25, Perking Elmer). Standard fluorescence quartz cuvettes were used for all measurements. Spectra of visible LEDs were recorded using an optical Spectrum Analyzer (Yokogawa AO6373B). The incident photon flux density was determined via a physical method using a High Sensitivity Thermopile sensor (Coherent) connected to a Laser Power/Energy Meter display (Coherent LabMax-Top). ${ }^{1} \mathrm{H}$ NMR spectra were recorded on a Bruker SB Avance III $300 \mathrm{MHz}$ spectrometer and on a Bruker SB Avance III HD $600 \mathrm{MHz}$. Chemical shifts are given in parts per million relative to solvent signal.

\section{Microfluidic set up}

Microphotoreactors were made from FEP tubing (i.d. $=0.8 \mathrm{~mm}, \mathrm{~S}_{181504}$, Cluzeau Info Labo (C.I.L.)). All syringes were connected through swagelok end fitting (stainless steel (C.I.L.)) and one piece fingertight fitting (10-32 PEEK, F-120X, (C.I.L.)) to the FEP tubing. Two different flow systems R1 (i.d. $=800 \mu \mathrm{m}, \mathrm{I}=21 \mathrm{~cm}, \mathrm{~V}=0.11 \mathrm{~mL}$ ) or R2 (i.d. $=800 \mu \mathrm{m}, \mathrm{I}=2.73 \mathrm{~m}, \mathrm{~V}=1.37$ $\mathrm{mL}$ ) were used during the experiments to perform kinetics studies. The different flow rates of the performed reactions were regulated using a Harvard Apparatus PHD ULTRA XF syringe pump (Holliston) fitted with 8 $\mathrm{mL}$ stainless steel syringes ( $\mathrm{PC}_{5}$ 702267, Harvard Apparatus). Microphoteactors were illuminated using either a $6 \mathrm{~W}$ mercury arc UVB lamp (Bioblock Scientific, $\lambda=312 \mathrm{~nm}$ ) or Blue $\left(\lambda=45^{8} \pm 25 \mathrm{~nm}\right)$ and green $(\lambda$ $=524 \pm 30 \mathrm{~nm}$ ) high power spots LEDs ( $50 \mathrm{~W}$ electrical power, 4500 lumens, 0.02 W. $\mathrm{cm}^{-2}$ ) from Bridgelux (Fig S1, $\mathrm{SI}$ ).

\section{Actinometry procedure with dithienylethene derivatives}

All syringes, connecting tubes and collecting round bottom flask were covered with aluminum foil to avoid the exposure of the actinometer solution to the ambient light. The first step consisted in preparing a suitable actinometric solution. The UV-Vis spectrum of the sample was measured prior to irradiation. Hence, a solution of the opened-ring isomer $1 \mathrm{a}\left(3.6 .10^{-5}\right.$ $\mathrm{M})$ or $\mathbf{2 a}$ in $n$-hexane $\left(1.8 .10^{-5} \mathrm{M}\right)$ was pump through the microphotoreactor (i.d. $=800 \mu \mathrm{m}, \mathrm{I}=21 \mathrm{~cm}, \mathrm{~V}=0.11 \mathrm{~mL}$ ) and irradiated with UV light at $312 \mathrm{~nm}$ during $9 \mathrm{~s}$ and $10 \mathrm{~s}$ respectively to reach the photostationary state (PSS). All the experimental runs were carried out at room temperature thanks to a module peltier system. Then, the resulting solution composed of a mixture 
of opened-ring and closed-ring isomers of diarylethenes $\mathbf{1}$ and $\mathbf{2}$ was injected into FEP tubing microreactors $\mathrm{R}_{1}$ or $\mathrm{R}_{2}$ and irradiated at the desired visible wavelength $(\lambda=458$ or $524 \mathrm{~nm})$ using various flow rates (between 0.2 and $12 \mathrm{~mL}$.min-1). After each irradiation period, absorption spectra were recorded at the excitation wavelength. Note that the reference solution should be kept in the dark and the whole procedure needs to be carried out in a dark room under red dimmed light.

General procedure for the photoisomerization of (E)-azobenzene

All syringes, connecting tubes and collecting round bottom flask were covered with aluminum foil to avoid the exposure of the actinometer solution to the ambient light. A solution of (E)-Azobenzene of concentration $0.01 \mathrm{M}$ in $n$-hexane was fed through FEP tubing microreactors $\mathrm{R} 1$ or $\mathrm{R} 2$ and irradiated at the desired visible wavelength $(\lambda=$ 458 or $524 \mathrm{~nm}$ ) at various flow rates (between 1 and $13 \mathrm{~mL} \cdot \mathrm{min}^{-1}$ ). The solvent of irradiated samples was evaporated at $20^{\circ} \mathrm{C}$ and the residue was then dissolved in $500 \mu \mathrm{L}$ of $\mathrm{CDCl}_{3}$ for analysis by ${ }^{1} \mathrm{H}$ NMR spectroscopy. The degree of conversion was calculated by integrating the peaks that correspond to the $(Z)$-Azobenzene and to the $(E)$-Azobenzene isomers in the ${ }^{1} \mathrm{H}$ spectrum. The obtained results were then used to determine quantum yield of $(E)$-azobenzene photoisomerisation $\left(\Phi_{E}\right)$. The same protocol was then performed in methanol and acetonitrile. Note, to ensure the stability of the NMR samples in $\mathrm{CDCl}_{3}$ over time, they should be kept in the dark.

\section{Supplementary Material}

Supporting information for this article is available on the WWW under http://dx.doi.org/10.1002/MS-number.

\section{Acknowledgements}

The authors gratefully acknowledge the CNRS, Lille University and ANR (ANR-19-CE07-0014) for the financial support. We are thankful for support from the NMR facility.

\section{Author Contribution Statement}

M. Roseau performed the experiments and analyzed the data. V. De Waele characterized the visible LEDs, X. Trivelli and F-X. Cantrelle performed the NMR analysis and M. Penhoat analyzed the data. L. Chausset-Boissarie conceived and designed the experiments, analyzed the data and wrote the paper.

\section{References}

[1] Q.-Q. Zhou, Y.-O. Zou, L.-Q. Lu, W.-J. Xiao, 'Visible-light-induced organic photochemical reactions through energy-transfer pathways', Angew. Chem., Int. Ed. 2019, 58, 1586-1604

[2] C. Michelin, N. Hoffmann, 'Photosensitization and PhotocatalysisPerspectives in Organic Synthesis', ACS Catal. 2018, 8, 12046-12055.

[3] J. Xie, H. Jin, A.S.K. Hashmi, 'The recent achievements of redox-neutral radical C-C cross-coupling enabled by visible-light', Chem. Soc. Rev. 2017, 46, 51935203
[4] J. Twilton, C. Le, P. Zhang, M.H. Shaw, R.W. Evans, D.W.C. MacMillan, 'The merger of transition metal and photocatalysis', Nat. Rev. Chem. 2017, 1, 0052

[5] M.H. Shaw, J. Twilton, D.W.C. MacMillan, 'Photoredox Catalysis in Organic Chemistry', J. Org. Chem. 2016, 81, 6898-6926

[6] N.A. Romero, D.A. Nicewicz, 'Organic Photoredox Catalysis', Chem. Rev. 2016, $116,10075-10166$

[7] M.D. Karkas, J.A. Porco, C.R.J. Stephenson, 'Photochemical Approaches to Complex Chemotypes: Applications in Natural Product Synthesis', Chem. Rev. 2016, 116, 9683-9747.

[8] F. Politano, G. Oksdath-Mansilla, 'Light on the Horizon: Current Research and Future Perspectives in Flow Photochemistry', Org. Process Res. Dev. 2018, 22, 1045-1062

[9] M.B. Plutschack, B. Pieber, K. Gilmore, P.H. Seeberger, 'The Hitchhiker's Guide to Flow Chemistry', Chem. Rev. 2017, 117, 11796-11893

[10] M. Oelgemoeller, T. Goodine, P. Malakar, 'Flow photochemistry - a green technology with a bright future', Wiley-VCH Verlag GmbH \& Co. KGaA, 2017, 1-24

[11] D. Cambie, C. Bottecchia, N.J.W. Straathof, V. Hessel, T. Noel, 'Applications of Continuous-Flow Photochemistry in Organic Synthesis, Material Science, and Water Treatment', Chem. Rev. 2016, 116, 10276-10341

[12] H.J. Kuhn, S.E. Braslavsky, R. Schmidt, 'Chemical actinometry' (IUPAC technical report), Pure Appl. Chem. 2004, 76, 2105-2146.

[13] B. Wriedt, D. Ziegenbalg, 'Common pitfalls in chemical actinometry', J Flow Chem 2020, 10, 295-306.

[14] T. Aillet, K. Loubiere, O. Dechy-Cabaret, L. Prat, 'Accurate measurement of the photon flux received inside two continuous flow microphotoreactors by actinometry', Int. J. Chem. React. Eng. 2014, 12, 1-13.

[15] C.G. Hatchard, C.A. Parker, 'A new sensitive chemical actinometer. II. Potassium ferrioxalate as a standard chemical actinometer', Proc. R. Soc. A 1956, 235, 518-536.

[16] B. Wriedt, D. Kowalczyk, D. Ziegenbalg, 'Experimental Determination of Photon Fluxes in Multilayer Capillary Photoreactors', ChemPhotoChem 2018, 2, 913-921

[17] R. Radjagobalou, J.-F. Blanco, V. Dias da Silva Freitas, C. Supplis, F. Gros, O. Dechy-Cabaret, K. Loubiere, 'A revised experimental protocol for implementing the actinometry method with the Reinecke's salt', J. Photochem. Photobiol., A 2019, 382, 111934

[18] J.-F. Cornet, A. Marty, J.-B. Gros, 'Revised technique for the determination of mean incident light fluxes on photobioreactors', Biotechnol. Prog. 1997, 13, 408-415.

[19] E.E. Wegner, A.W. Adamson, 'Photochemistry of complex ions. III. Absolute quantum yields for the photolysis of some aqueous chromium(III) complexes. Chemical actinometry in the long wavelength visible region', J. Am. Chem. Soc. 1966, 88, 394-404.

[20] H.D. Brauer, R. Schmidt, G. Gauglitz, S. Hubig, 'Chemical actinometry in the visible (475-610 nm) by meso-diphenylhelianthrene', Photochem. Photobiol. 1983, 37, 595-598.

[21] H.G. Heller, J.R. Langan, 'Photochromic heterocyclic fulgides. Part 3. The use of (E)- $\alpha$-(2,5-dimethyl-3-furylethylidene)(isopropylidene)succinic anhydride as a simple convenient chemical actinometer', J. Chem. Soc., Perkin Trans. 2 1981, 2, 341-343.

[22] M. Reinfelds, V. Hermanns, T. Halbritter, J. Wachtveitl, M. Braun, T. Slanina, A. Heckel, A Robust, 'Broadly Absorbing Fulgide Derivative as a Universal Chemical Actinometer for the UV to NIR Region', ChemPhotoChem 2019, 3, 441-449 
[23] Y. Yokoyama, K. Takahashi, 'Trifluoromethyl-substituted photochromic indolylfulgide. A remarkably durable fulgide towards photochemical and thermal treatments', Chem. Lett. 1996, 12, 1037-1038

[24] A. Roibu, S. Fransen, M.E. Leblebici, G. Meir, T. Van Gerven, S. Kuhn, 'An accessible visible-light actinometer for the determination of photon flux and optical pathlength in flow photo microreactors', Sci. Rep. 2018, 8, 1-10

[25] T. Sumi, Y. Takagi, A. Yagi, M. Morimoto, M. Irie, 'Photoirradiation wavelength dependence of cycloreversion quantum yields of diarylethenes', Chem. Commun. 2014, 50, 3928-3930

[26] K. Stranius, K. Boerjesson, 'Determining the Photoisomerization Quantum Yield of Photoswitchable Molecules in Solution and in the Solid State', Sci. Rep. 2017, 7, 41145 .

[27] V. Ladanyi, P. Dvorak, J. Al Anshori, L. Vetrakova, J. Wirz, D. Heger, 'Azobenzene photoisomerization quantum yields in methanol redetermined', Photochem. Photobiol. Sci. 2017, 16, 1757-1761

[28] G. Gauglitz, S. Hubig, 'Chemical actinometry in the UV by azobenzene in concentrated solution: a convenient method', J. Photochem. 1985, 30, 121-125.

[29] G. Gauglitz, S. Hubig, 'Azobenzene as a convenient actinometer: evaluation values for UV mercury lines and for the molecular nitrogen laser line', $J$. Photochem. 1981, 15, 255-257.

[30] G. Gauglitz, 'Azobenzene as a convenient actinometer for the determination of quantum yields of photoreactions', J. Photochem. 1976, 5, 41-47

[31] J. Ronayette, R. Arnaud, P. Lebourgeois, J. Lemaire, 'Photochemical isomerization of azobenzene in solution' Can. J. Chem. 1974, 52, 1848-1857

[32] S. Yamashita, H. Ono, O. Toyama, 'The cis-trans photoisomerization of azobenzene', Bull. Chem. Soc. Jpn. 1962, 35, 1849-1853

[33] G. Zimmerman, L.-Y. Chow, U.-J. Paik, 'The photochemical isomerization of azobenzene', J. Am. Chem. Soc. 1958, 80, 3528-3531.

[34] N. El Achi, Y. Bakkour, L. Chausset-Boissarie, M. Penhoat, C. Rolando, 'Rapid and facile chemical actinometric protocol for photo-microfluidic systems using azobenzene and NMR spectroscopy', RSC Adv. 2017, 7, 29815-29820.

[35] D. Russo, D. Spasiano, M. Vaccaro, R. Andreozzi, G. Li Puma, N.M. Reis, R. Marotta, 'Direct photolysis of benzoylecgonine under UV irradiation at $254 \mathrm{~nm}$ in a continuous flow microcapillary array photoreactor', Chem. Eng. J. 2016, 283, $243-250$ 


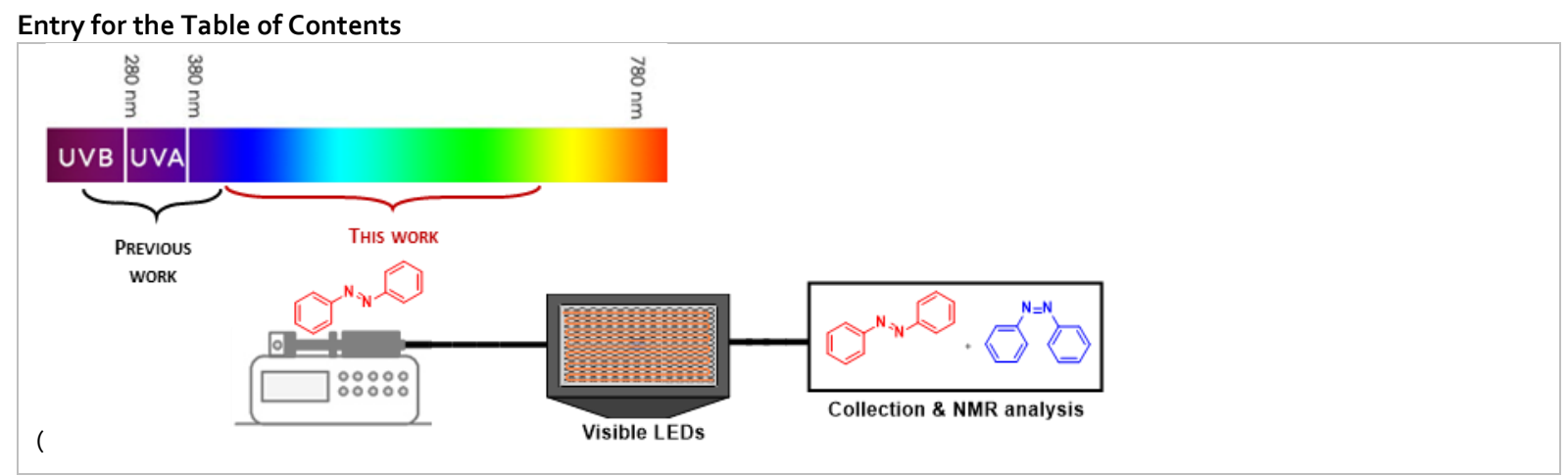

\section{Twitter}

The tweet text should not be more than 200 characters. Please describe your work with very short terms. 